\title{
Theory and Application of Characteristic Finite Difference Fractional Step Method of Capillary Force Enhanced Oil Production
}

\author{
Yirang Yuan ${ }^{1}$, Aijie Cheng ${ }^{1}$, Danping Yang ${ }^{1}$, Changfeng $\mathrm{Li}^{1,2} \&$ Tongjun Sun ${ }^{1}$ \\ ${ }^{1}$ Institute of Mathematics, Shandong University, Jinan, China \\ ${ }^{2}$ School of Economics, Shandong University, Jinan, China \\ Correspondence: Yirang Yuan, Institute of Mathematics, Shandong University, Jinan, ShandaNanlu 27, China. \\ Tel: 86-531-88364732. E-mail: yryuan@sdu.edu.cn
}

Received: March 14, 2015 Accepted: April 3, 2015 Online Published: May 8, 2015

doi:10.5539/jmr.v7n2p150

URL: http://dx.doi.org/10.5539/jmr.v7n2p150

The research is financed by the National Tackling Key Problems Program (Grant Nos. 2011ZX05011-004, 2011ZX 05052, 2005020069), Major State Basic Research Development Program of China (Grant No. G19990328), National Natural Science Foundation of China (Grant Nos. 11101244, 11271231, 10771124, 10372052) and Doctorate Foundation of the Ministry of Education of China (Grant No. 20030422047).

\begin{abstract}
A kind of second-order implicit characteristic fractional step finite difference method is presented in this paper for the numerical simulation coupled system of enhanced (chemical) oil production on consideration capillary force in porous media. Some techniques, such as the calculus of variations, energy analysis method, commutativity of the products of difference operators, decomposition of high-order difference operators and the theory of a priori estimates are introduced and an optimal order error estimates in $l^{2}$ norm is derived. This method has been applied successfully the numerical simulation of enhanced oil production in actual oilfields, and the simulation results are quite interesting and satisfactory.
\end{abstract}

Keywords: enhanced (chemical) oil production, three-dimensional porous coupled system on consideration capillary force, second-order implicit characteristic fractional step differences, optimal order $l^{2}$ estimates, application in actual oilfields

\section{Introduction}

\subsection{Background and Mathematical Description}

Massive residual crude oil remains in the reservoir after water-flooding exploiting because the constraint of capillary force prevents the motion and the undesired fluidity ratio between displacement phase and driven phase influences the flow slightly. Then it is more important to develop the displacement efficiency. A popular method is discussed by adding some chemical addition agents such as polymer, surfactant and alkali into the injected mixture, which can improve the flooding efficiency. The polymer can optimize the fluidity of displacement phase, modify the ratio with respect to driven phases, balance the leading edges well, weaken the inner porous layer, and increase the efficiency of displacement and the pressure gradient. Surfactant and alkali can decrease interfacial tensions of different phases, then make the bound oil move and gather (Ewing, et al., 1988; Yuan, 2013; Yuan, et al., 1998) 12 3 .

\footnotetext{
${ }^{1}$ Institute of Mathematics, Shandong University, Exploration Institute of Daqing Petroleum Administration. Research and application of the polymer flooding software (summary of "Eighth-Five" national key science and technology program, Grant No. 85-203-01-08), 1995.10.

${ }^{2}$ Institute of Mathematics, Shandong University, Exploration and development Institute of Daqing Petroleum Limited Liability Corporation. Modification of solving mathematical models of the polymer and improvement of reservoir description. 2006.

${ }^{3}$ Institute of Mathematics, Shandong University, Shengli Oilfield Branch, China Petroleum \& Chemical Corporation. Research on key technology of high temperature and high salinity chemical agent displacement, Chapter 4, §4.1 Numerical method, 83-106. 2011.3.
} 
In view of capillary force, and immiscible and incompressible flow, this paper discusses a second-order characteristic fractional step difference method for numerical simulation of enhanced (chemical) oil production in porous media, and gives the theoretical analysis. Based on the former mathematical and mechanical theory, the software is accomplished, applied in national oilfields such as Daqing Oilfield and Shengli Oilfield and give rise to outstanding benefits and social value.

The mathematical model is described by a nonlinear coupled system with initial-boundary values (Ewing, et al., 1988; Yuan, 2013; Yuan, et al., 1998) ${ }^{1,2,3}$ :

$$
\begin{gathered}
\frac{\partial}{\partial t}\left(\phi c_{o}\right)-\nabla \cdot\left(\kappa(X) \frac{\kappa_{\gamma_{o}}\left(c_{o}\right)}{\mu_{o}} \nabla p_{o}\right)=q_{o}, X=\left(x_{1}, x_{2}, x_{3}\right)^{T} \in \Omega, t \in J=(0, T], \\
\frac{\partial}{\partial t}\left(\phi c_{w}\right)-\nabla \cdot\left(\kappa(X) \frac{\kappa \gamma_{w}\left(c_{w}\right)}{\mu_{w}} \nabla p_{w}\right)=q_{w}, X \in \Omega, t \in J=(0, T], \\
\phi \frac{\partial}{\partial t}\left(c_{w} s_{\alpha}\right)+\nabla \cdot\left(s_{\alpha} \mathbf{u}-\phi c_{w} K_{\alpha} \nabla s_{\alpha}\right)=Q_{\alpha}\left(X, t, c_{w}, s_{\alpha}\right), X \in \Omega, t \in J, \alpha=1,2, \cdots, n_{c},
\end{gathered}
$$

where $\Omega$ is a bounded computational domain. Let the subscripts " $o$ " and " $w$ " respectively denote the parameters of oil phase and water phase. The notations $c_{l}, p_{l}, \kappa_{\gamma_{l}}\left(c_{l}\right), \mu_{l}$ and $q_{l}$ denote the concentration, the pressure, the relative permeability, the viscosity and the output quantity with respect to the $l$-phase, respectively. $\phi$ means the rock porosity, $\kappa(X)$ means the absolute permeability and $s_{\alpha}=s_{\alpha}(X, t)$ denotes the component concentration. The components denote sorts of chemical agents such as the polymer, surfactant, alkali and other ions, and the number is denoted by $n_{c} . \mathbf{u}$ is Darcy velocity, $K_{\alpha}=K_{\alpha}(X)$ is diffusion coefficient, and $Q_{\alpha}$ is source sink term related with the output. The rock void space is assumed to be full of water and oil, $c_{o}+c_{w}=1$. The the capillary force is dependent of the concentration $c$, defined by $p_{c}(c)=p_{o}-p_{w}$, where $c=c_{w}=1-c_{o}$.

The models (1) and (2) should be expressed by a normal form (Ewing, et al., 1988; Yuan, 2013). Let $\lambda(c)=$ $\frac{\kappa_{\gamma_{o}}(c)}{\mu_{o}}+\frac{\kappa_{\gamma_{w}}(c)}{\mu_{w}}$ denote the total migration rate of two-phase fluid, and let $\lambda_{l}(c)=\frac{\kappa_{\gamma_{l}}(c)}{\mu_{l} \lambda(c)}, l=o, w$ denote relative migration rate corresponding to water or oil. Applying Chavent transformation (Ewing, et al., 1988; Yuan, 2013; Douglas, 1983):

$$
p=\frac{p_{o}+p_{w}}{2}+\frac{1}{2} \int_{o}^{p_{c}}\left\{\lambda_{o}\left(p_{c}^{-1}(\xi)\right)-\lambda_{w}\left(p_{c}^{-1}(\xi)\right)\right\} d \xi .
$$

The flow equation is derived from (1) and (2),

$$
-\nabla \cdot(\kappa(X) \lambda(c) \nabla p)=q, X \in \Omega, t \in J=(0, T]
$$

where $q=q_{o}+q_{w}$. The concentration equation is derived from the difference of (1) and (2),

$$
\phi \frac{\partial c}{\partial t}-\nabla \cdot\left(\kappa \lambda \lambda_{o} \lambda_{w} p_{c}^{\prime} \nabla c\right)-\frac{1}{2} \nabla \cdot\left(\kappa \lambda\left(\lambda_{o}-\lambda_{w}\right) \nabla p\right)=\frac{1}{2}\left(q_{o}-q_{w}\right) .
$$

Let $\mathbf{u}=-\kappa(X) \lambda(c) \nabla p$, and note that the fact $\lambda_{o}-\lambda_{w}=2 \lambda_{o}-1$, we get

$$
\phi \frac{\partial c}{\partial t}+\nabla \cdot\left(\kappa \lambda \lambda_{o} \lambda_{w} p_{c}^{\prime} \nabla c\right)-\lambda_{o}^{\prime} \mathbf{u} \cdot \nabla c=\frac{1}{2}\left\{\left(q_{w}-\lambda_{w} q\right)-\left(q_{o}-\lambda_{o} q\right)\right\},
$$

where

$$
\begin{aligned}
& q_{w}=q \text { and } q_{o}=0, \text { if } q \geq 0 \text { (water injection well), } \\
& q_{w}=\lambda_{w} q \text { and } q_{o}=\lambda_{o} q, \text { if } q<0 \text { (oil production well). }
\end{aligned}
$$

(1) and (2) are rewritten as follows,

$$
\begin{gathered}
\nabla \cdot \mathbf{u}=q(X, t), \quad X \in \Omega, t \in J=(0, T], \\
\mathbf{u}=-\kappa(X) \lambda(c) \nabla p, \quad X \in \Omega, t \in J, \\
\phi \frac{\partial c}{\partial t}-\lambda^{\prime}(c) \mathbf{u} \nabla c+\nabla \cdot\left(\kappa(X) \lambda \lambda_{o} \lambda_{w} p_{c}^{\prime} \nabla c\right)= \begin{cases}\lambda_{o} q, & q \geq 0, \\
0, & q<0 .\end{cases}
\end{gathered}
$$

It is clear to restate (6) and (7) in a normalized formula,

$$
\begin{aligned}
& -\nabla \cdot(a(X, c) \nabla p)=q(X, t), X \in \Omega, t \in J=(0, T], \\
& \mathbf{u}=-a(X, c) \nabla p, \quad X \in \Omega, t \in J,
\end{aligned}
$$




$$
\phi \frac{\partial c}{\partial t}+b(c) \mathbf{u} \cdot \nabla c-\nabla \cdot(D(X, c) \nabla c)=g(X, t, c), X \in \Omega, t \in J,
$$

where $a(X, c)=\kappa(X) \lambda(c), b(c)=-\lambda^{\prime}(c), D(X, c)=-\kappa(X) \lambda \lambda_{o} \lambda_{w} p_{c}^{\prime}(c)$. It follows that $g(X, t, c)=\lambda_{o} q$ as $q \geq 0$, and it is true that $g(X, t, c)=0$ as $q<0$. Using (8), we can express (3) in a computational form

$$
\phi c \frac{\partial s_{\alpha}}{\partial t}+\mathbf{u} \cdot \nabla s_{\alpha}-\nabla \cdot\left(\phi c K_{\alpha} \nabla s_{\alpha}\right)=Q_{\alpha}-s_{\alpha}\left(q+\phi \frac{\partial c}{\partial t}\right), X \in \Omega, t \in J, \alpha=1,2, \cdots, n_{c},
$$

Two different boundary value conditions are considered in this paper.

(I) The boundary value condition for the constant pressure is given as follows

$$
\begin{aligned}
& p=e(X, t), X \in \partial \Omega, t \in J, \\
& c=r(X, t), X \in \partial \Omega, t \in J, \\
& s_{\alpha}=r_{\alpha}(X, t), X \in \partial \Omega, t \in J, \alpha=1,2, \cdots, n_{c},
\end{aligned}
$$

where $\partial \Omega$ denotes the outer boundary surface of $\Omega$.

(II) The boundary value condition for no permeation case is defined by

$$
\begin{aligned}
& \mathbf{u} \cdot \gamma=0, X \in \partial \Omega, t \in J \\
& D \nabla c \cdot \gamma=0, X \in \partial \Omega, t \in J \\
& K_{\alpha} \nabla s_{\alpha} \cdot \gamma=0, X \in \partial \Omega, t \in J, \alpha=1,2, \cdots, n_{c},
\end{aligned}
$$

where $\gamma$ denotes the outer normal unit vector. An additional condition should be introduced to determine the pressure $p$ in consideration of no permeation case, and it is defined by

$$
\int_{\Omega} p d X=0, \quad t \in J
$$

The compatibility condition is

$$
\int_{\Omega} q d X=0, \quad t \in J
$$

and the initial value condition is

$$
\begin{aligned}
& c(X, 0)=c_{0}(X), X \in \Omega, \\
& s_{\alpha}(X, 0)=s_{\alpha, 0}(X), X \in \Omega, \alpha=1,2, \cdots, n_{c} .
\end{aligned}
$$

\subsection{Relevant Development}

Introducing an assumption of periodic condition, Douglas, Ewing, Wheeler, Russell and other scholars presented characteristic finite difference method and characteristic finite element method to analyze a type of two dimensional incompressible two-phase displacement problems and gave theoretical error estimates (Douglas, 1981, 1983; Douglas, Russell, 1982; Ewing, Russell, Wheeler, 1984). A combination method was discussed by a whole consideration of the characteristic method and normal finite difference method or normal finite element method, which can reflect the hyperbolic nature of one-order part of convection-diffusion equation and decrease truncation error. This combination technique can also overcome numerical oscillation and dispersion, and can improve greatly the computational stability and accuracy. Douglas and other scholars presented a mathematical model of slight compression, numerical method and theoretical analysis for two-dimensional compressible displacement problem under periodic assumption and began a modern numerical model research (Douglas, Roberts, 1983; Yuan, 1992, 1993; Ewing, 1983). The authors dropped the period condition, gave a new modified characteristic finite difference algorithm and finite element algorithm, and derived optimal order error estimates in $L^{2}$-norm (Yuan, 1994, 1996, 1996; Axelsson, Gustafasson, 1979; Ewing, Lazarov, et al., 1994, 1996). In numerical simulation of modern oilfields exploration and development, the computation is of greatly huge-scale, three-dimensional region and long time interval consideration and the number of nodes maybe amount up to tens of thousands or even millions. It is impossible to solve this computation by using normal methods and a typical technique, fractional step method, is introduced in this paper (Peaceman, 1980; Douglas, Gunn, 1963, 1964; Marchuk, 1990). Though Douglas and Peaceman applied this technique into oil-water two phases displacement problem successfully (Peaceman, 1980), a type of substantial difficulty appeared in their theoretical analysis. Fourier method, considered in the proof of 
the stability and convergence by Douglas and Peaceman, was only applied in the constant-coefficient problems, that is to say Fourier method is not popularized in general problems with variable coefficients (Douglas, Gunn, 1963, 1964; Marchuk, 1990). Considering actual application, numerical stability and accuracy, the authors present one second-order characteristic fractional step finite difference method for three-dimensional incompressible and immiscible two-phase displacement coupled problem of enhanced oil production with consideration of capillary force. This algorithm can overcome numerical oscillation and dispersion, and can decrease the computational scale by decomposing three-dimensional problem into three successive one-dimensional subproblems. Using the calculus of variation, energy analysis method, commutativity of the products of difference operators, decomposition of high-order difference operators and the theory of a priori estimates, the authors give the second-order convergence result of accuracy and error estimates in $l^{2}$-norm, and successfully solve the international problem formulated by Douglas and Ewing. The method discussed in this paper has been applied in numerical simulation of enhanced oil production and gives a fundamental research in energy mathematics ${ }^{1,2,3}$.

\subsection{Hypotheses}

In general, the problem is positive definite,

$$
\begin{aligned}
& 0<a_{*} \leq a(c) \leq a^{*}, 0<\phi_{*} \leq \phi(X) \leq \phi^{*}, \\
& 0<D_{*} \leq D(X, c) \leq D^{*}, 0<K_{*} \leq K_{\alpha}(X) \leq K^{*}, \alpha=1,2, \cdots, n_{c}, \\
& \left|\frac{\partial a}{\partial c}(X, c)\right| \leq A^{*},
\end{aligned}
$$

where $a_{*}, a^{*}, \phi_{*}, \phi^{*}, D_{*}, D^{*} K_{*}, K^{*}$ and $A^{*}$ are positive constants. $b(c), g(c)$ and $Q_{\alpha}\left(c, s_{\alpha}\right)$ are Lipschitz continuous in the $\varepsilon_{0}$ neighbours of exact solutions.

Exact solutions of $(8) \sim(14)$ are assumed to be suitably smooth,

$$
p, c, s_{\alpha} \in L^{\infty}\left(W^{4, \infty}\right) \cap W^{1, \infty}\left(W^{1, \infty}\right), \quad \frac{\partial^{2} c}{\partial t^{2}}, \frac{\partial^{2} s_{\alpha}}{\partial t^{2}} \in L^{\infty}\left(L^{\infty}\right), \alpha=1,2, \cdots, n_{c} .
$$

In this paper $M$ and $\varepsilon$ express a generic positive constant and a generic positive small constant, respectively, and they have different meanings at different places.

\section{Second-order Implicit Characteristic Fractional Step Finite Difference Method}

Without loss of generality, the computational domain is taken as a rectangular cube $\Omega=\{[0,1]\}^{3}$ and the problem is assumed to be $\Omega$-periodic. Note that numerical simulation is considered for the flow of interior region and the boundary condition affects the flow weakly, so the periodic assumption is reasonable and related contents can be found in the references (Douglas, 1983; Douglas, Russell, 1982; Douglas, 1981; Ewing, Russell, Wheeler, 1984; Douglas, Roberts, 1983). Then The boundary value conditions of no permeation case can be dropped. Let the partition $\Omega_{h}$ replace the domain $\Omega$ and $\partial \Omega_{h}$ denotes the boundary. Let $h=1 / N$ denote the uniform spacial step in three-dimensional domain and the notations are defined by $X=\left(x_{1}, x_{2}, x_{3}\right)^{T}, X_{i j k}=(i h, j h, k h)^{T}, t^{n}=n \Delta t$, $W\left(X_{i j k}, t^{n}\right)=W_{i j k}^{n}$,

$$
A_{i+1 / 2, j k}^{n}=\left[a\left(X_{i j k}, C_{i j k}^{n}\right)+a\left(X_{i+1, j k}, C_{i+1, j k}^{n}\right)\right] / 2, \quad a_{i+1 / 2, j k}^{n}=\left[a\left(X_{i j k}, c_{i j k}^{n}\right)+a\left(X_{i+1, j k}, c_{i+1, j k}^{n}\right)\right] / 2,
$$

and $A_{i, j+1 / 2, k}^{n}, a_{i, j+1 / 2, k}^{n}, A_{i j, k+1 / 2}^{n}, a_{i j, k+1 / 2}^{n}$ are defined similarly. Let

$$
\begin{aligned}
& \delta_{\bar{x}_{1}}\left(A^{n} \delta_{x_{1}} P^{n+1}\right)_{i j k}=h^{-2}\left[A_{i+1 / 2, j k}^{n}\left(P_{i+1, j k}^{n+1}-P_{i j k}^{n+1}\right)-A_{i-1 / 2, j k}^{n}\left(P_{i j k}^{n+1}-P_{i-1, j k}^{n+1}\right)\right], \\
& \delta_{\bar{x}_{2}}\left(A^{n} \delta_{x_{2}} P^{n+1}\right)_{i j k}=h^{-2}\left[A_{i, j+1 / 2, k}^{n}\left(P_{i, j+1, k}^{n+1}-P_{i j k}^{n+1}\right)-A_{i, j-1 / 2, k}^{n}\left(P_{i j k}^{n+1}-P_{i, j-1, k}^{n+1}\right)\right], \\
& \delta_{\bar{x}_{3}}\left(A^{n} \delta_{x_{3}} P^{n+1}\right)_{i j k}=h^{-2}\left[A_{i j, k+1 / 2}^{n}\left(P_{i j, k+1}^{n+1}-P_{i j k}^{n+1}\right)-A_{i j, k-1 / 2}^{n}\left(P_{i j k}^{n+1}-P_{i j, k-1}^{n+1}\right)\right], \\
& \nabla_{h}\left(A^{n} \nabla_{h} P^{n+1}\right)_{i j k}=\delta_{\bar{x}_{1}}\left(A^{n} \delta_{x_{1}} P^{n+1}\right)_{i j k}+\delta_{\bar{x}_{2}}\left(A^{n} \delta_{x_{2}} P^{n+1}\right)_{i j k}+\delta_{\bar{x}_{3}}\left(A^{n} \delta_{x_{3}} P^{n+1}\right)_{i j k} .
\end{aligned}
$$

The flow equation (8) is discretized by

$$
\nabla_{h}\left(A^{n} \nabla_{h} P^{n+1}\right)_{i j k}=G_{i j k}=h^{-3} \iint_{X_{i j k}+Q_{h}} q\left(X, t^{n+1}\right) d x_{1} d x_{2} d x_{3}, 1 \leq i, j, k \leq N,
$$


where $Q_{h}$ denotes the cube of the side-length $h$ centered at the origin. Darcy velocity $\mathbf{U}^{\mathbf{n}+\mathbf{1}}=\left(U_{1}^{n+1}, U_{2}^{n+1}, U_{3}^{n+1}\right)^{T}$ is approximated as follows

$$
U_{1, i j k}^{n+1}=-\frac{1}{2}\left[A_{i+1 / 2, j k}^{n} \frac{P_{i+1, j k}^{n+1}-P_{i j k}^{n+1}}{h}+A_{i-1 / 2, j k}^{n} \frac{P_{i j k}^{n+1}-P_{i-1, j k}^{n+1}}{h}\right],
$$

and the other quantities $U_{2, i j k}^{n+1}, U_{3, i j k}^{n+1}$ are computed analogously.

The implicit characteristic fractional step algorithm is discussed to solve the saturation equation (9). The method of characteristics is applied in discretizing first order hyperbolic part of (9), which is consistent with the physical transportation of the flow along the direction of characteristics. The algorithm has high order of accuracy and great stability, and runs well in a large time step (Douglas, 1983, 1981; Douglas, Russell, 1982). Let $\psi(X, \mathbf{u})=$ $\left[\phi^{2}(X)+|\mathbf{u}|^{2}\right]^{1 / 2}, \partial / \partial \tau=\psi^{-1}\{\phi \partial / \partial t+\mathbf{u} \cdot \nabla\}$, and approximate the derivative along the characteristics by backward differences,

$$
\frac{\partial c^{n+1}}{\partial \tau} \approx \frac{c^{n+1}-c^{n}\left(X-\phi^{-1}(X) \mathbf{u}^{n+1}(X) \Delta t\right)}{\Delta t\left(1+\phi^{-2}(X)\left|\mathbf{u}^{n+1}(X)\right|^{2}\right)^{1 / 2}} .
$$

It remains to compute the values of the saturation $c^{n+1}$ at the $(n+1)$-th time step as $c^{n}$ given. Replacing the differential quotient by difference quotient, and decomposing the saturation equation (9) into the following form,

$$
\begin{aligned}
& \left(1-\frac{\Delta t}{\phi} \frac{\partial}{\partial x_{1}}\left(D\left(c^{n+1}\right) \frac{\partial}{\partial x_{1}}\right)\right)\left(1-\frac{\Delta t}{\phi} \frac{\partial}{\partial x_{2}}\left(D\left(c^{n+1}\right) \frac{\partial}{\partial x_{2}}\right)\right)\left(1-\frac{\Delta t}{\phi} \frac{\partial}{\partial x_{3}}\left(D\left(c^{n+1}\right) \frac{\partial}{\partial x_{3}}\right)\right) c^{n+1} \\
& =\bar{c}^{n}+\frac{\Delta t}{\phi} f\left(X, t, c^{n+1}\right)+O\left((\Delta t)^{2}\right),
\end{aligned}
$$

where $\mathbf{u}=\left(u_{1}, u_{2}, u_{3}\right)^{T}$ and $\bar{c}^{n}=c\left(X-\phi^{-1}(X) b\left(c^{n+1}\right) \mathbf{u}^{n+1}(X) \Delta t, t^{n}\right)$. Then the second-order implicit characteristic finite difference program combined with fractional step is illustrated as follows,

$$
\begin{gathered}
\left(\phi-\Delta t \delta_{\bar{x}_{1}}\left(D\left(C^{n}\right) \delta_{x_{1}}\right)\right)_{i j k} C_{i j k}^{n+1 / 3}=\phi_{i j k} C_{i j k}^{n}+\Delta t f\left(X, t^{n}, C^{n}\right)_{i j k}, 1 \leq i \leq N . \\
\left(\phi-\Delta t \delta_{\bar{x}_{2}}\left(D\left(C^{n}\right) \delta_{x_{2}}\right)\right)_{i j k} C_{i j k}^{n+2 / 3}=\phi_{i j k} C_{i j k}^{n+1 / 3}, 1 \leq j \leq N . \\
\left(\phi-\Delta t \delta_{\bar{x}_{3}}\left(D\left(C^{n}\right) \delta_{x_{3}}\right)\right)_{i j k} C_{i j k}^{n+1}=\phi_{i j k} C_{i j k}^{n+2 / 3}, 1 \leq k \leq N .
\end{gathered}
$$

The discrete value of saturation $C^{n}(X)$ is defined by a piecewise threefold quadratic interpolation of the values of nodes $\left\{C_{i j k}^{n}\right\}$, and other symbols are defined by $\hat{C}_{i j k}^{n}=C^{n}\left(\hat{X}_{i j k}^{n}\right), \hat{X}_{i j k}^{n}=X_{i j k}-\phi_{i j k}^{-1} b\left(C_{i j k}^{n}\right) \mathbf{U}_{i j k}^{n+1} \Delta t$.

Introducing the symbols $\hat{\phi}^{n+1}=\phi C^{n+1}, \hat{\phi}^{n+1,-1}=\left(\hat{\phi}^{n+1}\right)^{-1}$, and $\hat{D}_{\alpha}^{n+1}=\phi C^{n+1} K_{\alpha}$, we can approximate the concentration equation (10) by an implicit characteristic fractional step finite difference parallel algorithm,

$$
\begin{gathered}
\left(\hat{\phi}^{n+1}-\Delta t \delta_{\bar{x}_{1}}\left(\hat{D}_{\alpha}^{n+1} \delta_{x_{1}}\right)\right)_{i j k} S_{\alpha, i j k}^{n+1 / 3}=\hat{\phi}_{i j k}^{n+1} \hat{S}_{\alpha, i j k}^{n}+\Delta t\left\{Q_{\alpha}\left(C_{i j k}^{n+1}, S_{\alpha, i j k}^{n}\right)\right. \\
\left.-S_{\alpha, i j k}^{n}\left(q\left(C_{i j k}^{n+1}\right)+\phi_{i j k} \frac{C_{i j k}^{n+1}-C_{i j k}^{n}}{\Delta t}\right)\right\}, 1 \leq i \leq N, \alpha=1,2, \cdots, n_{c}, \\
\left(\hat{\phi}^{n+1}-\Delta t \delta_{\bar{x}_{2}}\left(\hat{D}_{\alpha}^{n+1} \delta_{x_{2}}\right)\right)_{i j k} S_{\alpha, i j k}^{n+2 / 3}=\hat{\phi}_{i j k}^{n+1} S_{\alpha, i j k}^{n+1 / 3}, 1 \leq j \leq N, \alpha=1,2, \cdots, n_{c}, \\
\left(\hat{\phi}^{n+1}-\Delta t \delta_{\bar{x}_{3}}\left(\hat{D}_{\alpha}^{n+1} \delta_{x_{3}}\right)\right)_{i j k} S_{\alpha, i j k}^{n+1}=\hat{\phi}_{i j k}^{n+1} S_{\alpha, i j k}^{n+2 / 3}, 1 \leq k \leq N, \alpha=1,2, \cdots, n_{c},
\end{gathered}
$$

where $\hat{S}_{\alpha}^{n}(X)\left(\alpha=1,2, \cdots, n_{c}-1\right)$ is computed by a piecewise threefold quadratic interpolation of the values of neighbour nodes $\left\{S_{\alpha, i j k}^{n}\right\}$, and $\hat{S}_{\alpha, i j k}^{n}=S_{\alpha}^{n}\left(\hat{X}_{i j k}^{n}\right), \hat{X}_{i j k}^{n}=X_{i j k}-\hat{\phi}_{i j k}^{n+1,-1} \mathbf{U}_{i j k}^{n+1} \Delta t$.

Initial value conditions:

$$
P_{i j k}^{0}=p_{0}\left(X_{i j k}\right), C_{i j k}^{0}=c_{0}\left(X_{i j k}\right), S_{\alpha, i j k}^{0}=s_{\alpha, 0}\left(X_{i j k}\right), X_{i j k} \in \Omega_{h}, \alpha=1,2, \cdots, n_{c} .
$$

The implicit program runs in the following order. Given $\left\{P_{i j k}^{n}, C_{i j k}^{n}, S_{\alpha, i j k}^{n}, \alpha=1,2, \cdots, n_{c}\right\}$, the pressure $\left\{P_{i j k}^{n+1}\right\}$ is computed by elimination method or conjugate gradient method from the difference equation (17). Then the values of Darcy velocity $\left\{\mathbf{U}_{i j k}^{n+1}\right\}$ are obtained by (18). The solution of transition sheaf $\left\{C_{i j k}^{n+1 / 3}\right\}$ in $x_{1}$ direction is computed 
by the method of speedup from (20), then $\left\{C_{i j k}^{n+2 / 3}\right\}$ proceeds in $x_{2}$ direction by $(21),\left\{C_{i j k}^{n+1}\right\}$ is obtained finally in $x_{3}$ direction by (22) similarly. Then the computation of the saturation goes on. The saturation $\left\{S_{\alpha, i j k}^{n+1}\right\}$ is given by (25) after $\left\{S_{\alpha, i j k}^{n+1 / 3}\right\},\left\{S_{\alpha, i j k}^{n+2 / 3}\right\}$ are computed respectively in $x_{1}, x_{2}$ directions by the method of speedup from (23) and (24) as shown in the above expression. It is accomplished in parallel with respect to $\alpha=1,2, \cdots, n_{c}$. The solutions of (17), (20) (22), (23) (25) and (26) exist and are sole because of the positive definite condition.

\section{Convergence Analysis}

Let $\pi=p-P, \xi=c-C, \zeta_{\alpha}=s_{\alpha}-S_{\alpha}$, where $p, c$ and $s_{\alpha}$ are exact solutions of (8) (13), and $P, C$ and $S_{\alpha}$ are discrete solutions of $(17) \sim(26)$. Introduce the inner products and norms in discrete space $l^{2}(\Omega)$ (Yuan, 2010, 2012).

$$
<f, g>=\sum_{i, j, k=1}^{N} f_{i j k} g_{i j k} h^{3},\|f\|_{0}^{2}=<f, f>.
$$

$<D \nabla_{h} f, \nabla_{h} f>$ denotes the square of weighted semi-norm in $h^{1}(\Omega)$ corresponding to the space $H^{1}(\Omega)=W^{1,2}(\Omega)$, and $D(X)$ is positive definite.

Theorem 1 Assume that exact solutions of (8) (13) are appropriately smooth, $p, c \in W^{1, \infty}\left(W^{1, \infty}\right) \cap L^{\infty}\left(W^{4, \infty}\right)$, $s_{\alpha} \in W^{1, \infty}\left(W^{1, \infty}\right) \cap L^{\infty}\left(W^{4, \infty}\right), \frac{\partial c}{\partial \tau} \in L^{\infty}\left(W^{4, \infty}\right), \frac{\partial s_{\alpha}}{\partial \tau_{\alpha}} \in L^{\infty}\left(W^{4, \infty}\right), \frac{\partial^{2} c}{\partial \tau^{2}}, \frac{\partial^{2} s_{\alpha}}{\partial \tau_{\alpha}^{2}} \in L^{\infty}\left(L^{\infty}\right), \alpha=1,2, \cdots, n_{c}$. The modified characteristics method is applied to compute (17) (25) layer by layer, and the partition parameters satisfy

$$
\Delta t=O\left(h^{2}\right)
$$

then the following error estimates hold

$$
\begin{aligned}
& \|p-P\|_{\bar{L}^{\infty}\left((0, T] ; h^{1}\right)}+\|c-C\|_{\bar{L}^{\infty}\left((0, T] ; l^{2}\right)}+\|c-C\|_{\bar{L}^{2}\left((0, T] ; h^{1}\right)} \leq M_{1}^{*}\left\{\Delta t+h^{2}\right\}, \\
& \left\|s_{\alpha}-S_{\alpha}\right\|_{\bar{L}^{\infty}\left((0, T] ; l^{2}\right)}+\left\|s_{\alpha}-S_{\alpha}\right\|_{\bar{L}^{2}\left((0, T] ; h^{1}\right)} \leq M_{2}^{*}\left\{\Delta t+h^{2}\right\}, \alpha=1,2, \cdots, n_{c},
\end{aligned}
$$

where $\|g\|_{\bar{L}^{\infty}(J ; \mathcal{M})}=\sup _{n \Delta t \leq T}\left\|g^{n}\right\|_{\mathcal{M}}$, and $M_{1}^{*}=M_{1}^{*}\left(\|p\|_{W^{1, \infty}\left(W^{4, \infty}\right)},\|p\|_{L^{\infty}\left(W^{4, \infty}\right)},\|c\|_{W^{1, \infty}\left(W^{4, \infty}\right)},\left\|\frac{\partial c}{\partial \tau}\right\|_{L^{\infty}\left(W^{4, \infty}\right)},\left\|\frac{\partial^{2} c}{\partial \tau^{2}}\right\|_{L^{\infty}\left(L^{\infty}\right)}\right)$, $M_{2}^{*}=M_{2}^{*}\left(\left\|s_{\alpha}\right\|_{W^{1, \infty}\left(W^{4, \infty}\right)},\left\|\frac{\partial s_{\alpha}}{\partial \tau_{\alpha}}\right\|_{L^{\infty}\left(W^{4, \infty}\right)},\left\|\frac{\partial^{2} s_{\alpha}}{\partial \tau_{\alpha}^{2}}\right\|_{L^{\infty}\left(L^{\infty}\right)}\right)$

Proof. The error equation of the pressure is derived from the difference of $(8)\left(t=t^{n+1}\right)$ and (17),

$$
-\nabla_{h}\left(A^{n} \nabla_{h} \pi^{n+1}\right)_{i j k}=\nabla_{h}\left(\left[a\left(c^{n+1}\right)-a\left(c^{n}\right)\right] \nabla_{h} p^{n+1}\right)_{i j k}+\sigma_{i j k}^{n+1}, 1 \leq i, j, k \leq N,
$$

where $\left|\sigma_{i j k}^{n+1}\right| \leq M\left(\|p\|_{L^{\infty}\left(W^{4, \infty}\right)},\|c\|_{L^{\infty}\left(W^{3, \infty}\right)}\right)\left\{\Delta t+h^{2}\right\}$.

Testing both sides of (29) by $\pi^{n+1}$, and summing by parts

$$
\left\langle A^{n} \nabla_{h} \pi^{n+1}, \nabla_{h} \pi^{n+1}\right\rangle=\left\langle\sigma^{n+1}, \pi^{n+1}\right\rangle-\left\langle\left[a\left(c^{n+1}\right)-a\left(c^{n}\right)\right] \nabla_{h} p^{n+1}, \nabla_{h} \pi^{n+1}\right\rangle .
$$

Then,

$$
\left\|\nabla_{h} \pi^{n+1}\right\| \leq M\left(\left\|p^{n+1}\right\|_{4, \infty},\left\|c^{n+1}\right\|_{3, \infty}\right)\left\{\left\|\xi^{n}\right\|+h^{2}+\Delta t\right\}
$$

The error of the saturation is estimated later. Canceling the transient terms $C_{i j k}^{n+1 / 3}$ and $C_{i j k}^{n+2 / 3}$ in (20), (21) and (22), introducing $\overline{\mathbf{U}}^{n+1}=b\left(C^{n}\right) \mathbf{U}^{n+1}$, and simplifying the dispersion term $D\left(C^{n}\right) \approx D(X)$ for convenience (this simplification does not affect the nature of numerical analysis, that is to say the following process can be applied in the theoretical analysis related of $D\left(C^{n}\right)$ ), we can obtain an equivalent difference equation,

$$
\begin{aligned}
& \phi_{i j k} \frac{C_{i j k}^{n+1}-\hat{C}_{i j k}^{n}}{\Delta t}-\sum_{\beta=1}^{3} \delta_{\bar{x}_{\beta}}\left(D \delta_{x_{\beta}} C^{n+1}\right)_{i j k} \\
& =g\left(X_{i j k}, t^{n}, C_{i j k}^{n}\right)-\Delta t\left\{\delta_{\bar{x}_{1}}\left(D \delta_{x_{1}}\left(\phi^{-1} \delta_{\bar{x}_{2}}\left(D \delta_{x_{2}} C^{n+1}\right)\right)\right)_{i j k}+\delta_{\bar{x}_{1}}\left(D \delta_{x_{1}}\left(\phi^{-1} \delta_{\bar{x}_{3}}\left(D \delta_{x_{3}} C^{n+1}\right)\right)\right)_{i j k}\right. \\
& \left.+\delta_{\bar{x}_{2}}\left(D \delta_{x_{2}}\left(\phi^{-1} \delta_{\bar{x}_{3}}\left(D \delta_{x_{3}} C^{n+1}\right)\right)\right)_{i j k}\right\}+(\Delta t)^{2} \delta_{\bar{x}_{1}}\left(D \delta_{x_{1}}\left(\phi^{-1} \delta_{\bar{x}_{2}}\left(D \delta_{x_{2}}\left(\phi^{-1} \delta_{\bar{x}_{3}}\left(D \delta_{x_{3}} C^{n+1}\right)\right)\right)\right)\right)_{i j k}, 1 \leq i, j, k \leq N .
\end{aligned}
$$


Let $\widetilde{\mathbf{u}}^{n+1}=b\left(c^{n+1}\right) \mathbf{u}^{n+1}$, and we derive error equation of the saturation from (9) $\left(t=t^{n+1}\right)$ and (32),

$$
\begin{aligned}
& \phi_{i j k} \frac{\xi_{i j k}^{n+1}-\left(c\left(\bar{X}_{i j k}^{n}\right)-\hat{C}_{i j k}^{n}\right)}{\Delta t}-\sum_{\beta=1}^{3} \delta_{\bar{x}_{\beta}}\left(D \delta_{x_{\beta}} C^{n+1}\right)_{i j k} \\
& =g\left(X_{i j k}, t^{n+1}, c_{i j k}^{n+1}\right)-g\left(X_{i j k}, t^{n}, C_{i j k}^{n}\right)-\Delta t\left\{\delta_{\bar{x}_{1}}\left(D \delta_{x_{1}}\left(\phi^{-1} \delta_{\bar{x}_{2}}\left(D \delta_{x_{2}} \xi^{n+1}\right)\right)\right)_{i j k}\right. \\
& \left.+\delta_{\bar{x}_{1}}\left(D \delta_{x_{1}}\left(\phi^{-1} \delta_{\bar{x}_{2}}\left(D \delta_{x_{2}} \xi^{n+1}\right)\right)\right)_{i j k}+\delta_{\bar{x}_{2}}\left(D \delta_{x_{2}}\left(\phi^{-1} \delta_{\bar{x}_{3}}\left(D \delta_{x_{3}} \xi^{n+1}\right)\right)\right)_{i j k}\right\} \\
& +(\Delta t)^{2} \delta_{\bar{x}_{1}}\left(D \delta_{x_{1}}\left(\phi^{-1} \delta_{\bar{x}_{2}}\left(D \delta_{x_{2}}\left(\phi^{-1} \delta_{\bar{x}_{3}}\left(D \delta_{x_{3}} \xi^{n+1}\right)\right)\right)\right)\right)_{i j k}+\varepsilon_{i j k}^{n+1}, 1 \leq i, j, k \leq N,
\end{aligned}
$$

where $\bar{X}_{i j k}^{n}=X_{i j k}-\phi_{i j k}^{-1} \overline{\mathbf{u}}_{i j k}^{n+1} \Delta t,\left|\varepsilon_{i j k}^{n+1}\right| \leq M\left(\left\|\frac{\partial^{2} c}{\partial \tau^{2}}\right\|_{L^{\infty}\left(L^{\infty}\right)},\left\|\frac{\partial c}{\partial \tau}\right\|_{L^{\infty}\left(W^{4, \infty}\right)},\|c\|_{L^{\infty}\left(W^{4, \infty}\right)}\right)\left\{h^{2}+\Delta t\right\}$.

Multiplying the both sides of (33) by $\xi_{i j k}^{n+1} \Delta t$, summing by parts and rewriting the result in inner products,

$$
\begin{aligned}
& \left\langle\phi \frac{\xi^{n+1}-\hat{\xi}^{n}}{\Delta t}, \xi^{n+1}\right\rangle \Delta t+\sum_{\beta=1}^{3}\left\langle D \delta_{x_{\beta}} \xi^{n+1}, \delta_{x_{\beta}} \xi^{n+1}\right\rangle \Delta t \\
& \leq M\left\{\left\|\xi^{n}\right\|^{2}+\left\|\xi^{n+1}\right\|^{2}+\left\|\nabla_{h} \pi^{n+1}\right\|^{2}+h^{4}+(\Delta t)^{2}\right\} \Delta t \\
& -(\Delta t)^{2}\left\{\left\langle\delta_{\bar{x}_{1}}\left(D \delta_{x_{1}}\left(\phi^{-1} \delta_{\bar{x}_{2}}\left(D \delta_{x_{2}} \xi^{n+1}\right)\right)\right), \xi^{n+1}\right\rangle+\cdots+\left\langle\delta_{\bar{x}_{2}}\left(D \delta_{x_{2}}\left(\phi^{-1} \delta_{\bar{x}_{3}}\left(D \delta_{x_{3}} \xi^{n+1}\right)\right)\right), \xi^{n+1}\right\rangle\right\} \\
& +(\Delta t)^{3}\left\langle\delta_{\bar{x}_{1}}\left(D \delta_{x_{1}}\left(\phi^{-1} \delta_{\bar{x}_{2}}\left(D \delta_{x_{2}}\left(\phi^{-1} \delta_{\bar{x}_{3}}\left(D \delta_{x_{3}} \xi^{n+1}\right)\right)\right)\right)\right), \xi^{n+1}\right\rangle .
\end{aligned}
$$

Introduce an induction hypothesis,

$$
\sup _{1 \leq n \leq L} \max \left\{\left\|\pi^{n}\right\|_{0, \infty},\left\|\xi^{n}\right\|_{0, \infty}\right\} \rightarrow 0, \quad(h, \Delta t) \rightarrow 0 .
$$

Using (35) and (31), we can obtain the following estimates from (34)

$$
\begin{aligned}
& \left\|\phi^{1 / 2} \xi^{n+1}\right\|^{2}-\left\|\phi^{1 / 2} \xi^{n}\right\|^{2}+\sum_{\beta=1}^{3}\left\langle D \delta_{x_{\beta}} \xi^{n+1}, \delta_{x_{\beta}} \xi^{n+1}\right\rangle \Delta t \\
& \leq \varepsilon\left\|\nabla_{h} \xi^{n+1}\right\|^{2} \Delta t+M\left\{\left\|\xi^{n}\right\|^{2}+\left\|\xi^{n+1}\right\|^{2}+h^{4}+(\Delta t)^{2}\right\} \Delta t \\
& -(\Delta t)^{2}\left\{\left\langle\delta_{\bar{x}_{1}}\left(D \delta_{x_{1}}\left(\phi^{-1} \delta_{\bar{x}_{2}}\left(D \delta_{x_{2}} \xi^{n+1}\right)\right)\right), \xi^{n+1}\right\rangle+\cdots+\left\langle\delta_{\bar{x}_{2}}\left(D \delta_{x_{2}}\left(\phi^{-1} \delta_{\bar{x}_{3}}\left(D \delta_{x_{3}} \xi^{n+1}\right)\right)\right), \xi^{n+1}\right\rangle\right\} \\
& +(\Delta t)^{3}\left\langle\delta_{\bar{x}_{1}}\left(D \delta_{x_{1}}\left(\phi^{-1} \delta_{\bar{x}_{2}}\left(D \delta_{x_{2}}\left(\phi^{-1} \delta_{\bar{x}_{3}}\left(D \delta_{x_{3}} \xi^{n+1}\right)\right)\right)\right)\right), \xi^{n+1}\right\rangle,
\end{aligned}
$$

where $\left\|\nabla_{h} \xi^{n+1}\right\|^{2}=\sum_{\beta=1}^{3}\left\|\delta_{x_{\beta}} \xi^{n+1}\right\|^{2}$.

The third and forth terms on the right side of (36) are considered. Though $-\delta_{\bar{x}_{1}}\left(D \delta_{x_{1}}\right),-\delta_{\bar{x}_{2}}\left(D \delta_{x_{2}}\right), \cdots$ are selfconjugate, positive definite and bounded operators, and the spacial domain is a unit cube, however their products are generally incommutative. Using the product commutativity of difference operators $\delta_{x_{1}} \delta_{x_{2}}=\delta_{x_{2}} \delta_{x_{1}}, \delta_{\bar{x}_{1}} \delta_{x_{2}}=\delta_{x_{2}} \delta_{\bar{x}_{1}}$, $\delta_{x_{1}} \delta_{\bar{x}_{2}}=\delta_{\bar{x}_{2}} \delta_{x_{1}}, \delta_{\bar{x}_{1}} \delta_{\bar{x}_{2}}=\delta_{\bar{x}_{2}} \delta_{\bar{x}_{1}}$, we can express the first part of the third term as follows

$$
\begin{aligned}
- & (\Delta t)^{2}\left\langle\delta_{\bar{x}_{1}}\left(D \delta_{x_{1}}\left(\phi^{-1} \delta_{\bar{x}_{2}}\left(D \delta_{x_{2}} \xi^{n+1}\right)\right)\right), \xi^{n+1}\right\rangle=(\Delta t)^{2}\left\langle\delta_{x_{1}}\left(\phi^{-1} \delta_{x_{2}}\left(D \delta_{x_{1}} \xi^{n+1}\right)\right), D \delta_{x_{1}} \xi^{n+1}\right\rangle \\
= & (\Delta t)^{2}\left\langle\delta_{x_{1}} \phi^{-1} \cdot \delta_{\bar{x}_{2}}\left(D \delta_{x_{2}} \xi^{n+1}\right)+\phi^{-1} \delta_{\bar{x}_{2}} \delta_{x_{1}}\left(D \delta_{x_{2}} \xi^{n+1}\right), D \delta_{x_{1}} \xi^{n+1}\right\rangle \\
= & (\Delta t)^{2}\left\{\left\langle\delta_{\bar{x}_{2}}\left(D \delta_{x_{2}} \xi^{n+1}, \delta_{x_{1}} \phi^{-1} D \delta_{x_{1}} \xi^{n+1}\right\rangle+\left\langle\delta_{\bar{x}_{2}} \delta_{x_{1}}\left(D \delta_{x_{2}} \xi^{n+1}\right), \phi^{-1} D \delta_{x_{1}} \xi^{n+1}\right\rangle\right\}\right. \\
= & -(\Delta t)^{2}\left\{\left\langle D \delta_{x_{2}} \xi^{n+1}, \delta_{x_{2}}\left(\delta_{x_{1}} \phi^{-1} D \delta_{x_{1}} \xi^{n+1}\right)\right\rangle+\left\langle\delta_{x_{1}}\left(D \delta_{x_{2}} \xi^{n+1}\right), \delta_{x_{2}}\left(\phi^{-1} D \delta_{x_{1}} \xi^{n+1}\right)\right\rangle\right\} \\
= & -(\Delta t)^{2}\left\{\left\langle D \delta_{x_{2}} \xi^{n+1}, \delta_{x_{2}}\left(\delta_{x_{1}} \phi^{-1} D\right) \delta_{x_{1}} \xi^{n+1}+\delta_{x_{1}} \phi^{-1} D \delta_{x_{1}} \delta_{x_{2}} \xi^{n+1}\right\rangle\right. \\
& \left.+\left\langle D \delta_{x_{1}} \delta_{x_{2}} \xi^{n+1}+\delta_{x_{1}} D \cdot \delta_{x_{2}} \xi^{n+1}, \phi^{-1} D \delta_{x_{1}} \delta_{x_{2}} \xi^{n+1}+\delta_{x_{2}}\left(\phi^{-1} D\right) \delta_{x_{1}} \xi^{n+1}\right\rangle\right\} \\
= & -(\Delta t)^{2}\left\{\left\langle D \delta_{x_{1}} \delta_{x_{2}} \xi^{n+1}, \phi^{-1} D \delta_{x_{1}} \delta_{x_{2}} \xi^{n+1}\right\rangle+\left\langle D \delta_{x_{1}} \delta_{x_{2}} \xi^{n+1}, \delta_{x_{2}}\left(\phi^{-1} D\right) \delta_{x_{1}} \xi^{n+1}+\delta_{x_{1}} \phi^{-1} \cdot D \delta_{x_{2}} \xi^{n+1}\right\rangle\right. \\
& \left.+\left\langle D \delta_{x_{2}} \xi^{n+1}, \delta_{x_{2}}\left(\delta_{x_{1}} \phi^{-1} D\right) \cdot \delta_{x_{1}} \xi^{n+1}\right\rangle+\left\langle\delta_{x_{1}} D \cdot \delta_{x_{2}} \xi^{n+1}, \delta_{x_{2}}\left(\phi^{-1} D\right) \cdot \delta_{x_{1}} \xi^{n+1}\right\rangle\right\} .
\end{aligned}
$$

Considering the first term on the right side of (37),

$$
-(\Delta t)^{2}\left\langle D \delta_{x_{1}} \delta_{x_{2}} \xi^{n+1}, \phi^{-1} D \delta_{x_{1}} \delta_{x_{2}} \xi^{n+1}\right\rangle \leq-(\Delta t)^{2} D_{*}^{2}\left(\phi^{*}\right)^{-1}\left\|\delta_{x_{1}} \delta_{x_{2}} \xi^{n+1}\right\|^{2} .
$$


Applying $\varepsilon$-Cauchy formula and cancelling the terms of $\delta_{x_{1}} \delta_{x_{2}} \xi^{n+1}$,

$$
\begin{aligned}
& -(\Delta t)^{2}\left\{\left\langle D \delta_{x_{1}} \delta_{x_{2}} \xi^{n+1}, \delta_{x_{2}}\left(\phi^{-1} D\right) \delta_{x_{1}} \xi^{n+1}+\delta_{x_{1}} \phi^{-1} \cdot D \delta_{x_{2}} \xi^{n+1}\right\rangle+\cdots\right\} \\
& \leq-\frac{(\Delta t)^{2}}{2} D_{*}^{2}\left(\phi^{*}\right)^{-1}\left\|\delta_{x_{1}} \delta_{x_{2}} \xi^{n+1}\right\|^{2}+M(\Delta t)^{2}\left\{\left\|\delta_{x_{1}} \xi^{n+1}\right\|^{2}+\left\|\delta_{x_{2}} \xi^{n+1}\right\|^{2}\right\} .
\end{aligned}
$$

Collecting (38), (27) and inverse estimates, we have the estimates of (37),

$$
\begin{aligned}
& -(\Delta t)^{2}\left\langle\delta_{\bar{x}_{1}}\left(D \delta_{x_{1}}\left(\phi^{-1} \delta_{\bar{x}_{2}}\left(D \delta_{x_{2}} \xi^{n+1}\right)\right)\right), \xi^{n+1}\right\rangle \\
& \leq M(\Delta t)^{2}\left\{\left\|\delta_{x_{1}} \xi^{n+1}\right\|^{2}+\left\|\delta_{x_{2}} \xi^{n+1}\right\|^{2}\right\} \\
& \leq M \Delta t \cdot h^{2}\left\{\left\|\delta_{x_{1}} \xi^{n+1}\right\|^{2}+\left\|\delta_{x_{2}} \xi^{n+1}\right\|^{2}\right\} \leq M\left\|\xi^{n+1}\right\|^{2} \Delta t .
\end{aligned}
$$

The other terms of the third term of (36) can be estimated similarly.

Summing by parts three times successively, extracting high-order terms of $\delta_{x_{1}} \delta_{x_{2}} \delta_{x_{3}} \xi^{n+1}$, cancelling them by $\varepsilon$-Cauchy formula and using the constraint condition (27) and inverse estimates, we can give the estimates of the forth term of (36)

$$
(\Delta t)^{3}\left\langle\delta_{\bar{x}_{1}}\left(D \delta_{x_{1}}\left(\phi^{-1} \delta_{\bar{x}_{2}}\left(D \delta_{x_{2}}\left(\phi^{-1} \delta_{\bar{x}_{3}}\left(D \delta_{x_{3}} \xi^{n+1}\right)\right)\right)\right)\right), \xi^{n+1}\right\rangle \leq M\left\|\xi^{n+1}\right\|^{2} \Delta t
$$

The estimates of (34) are given by a summary of the condition (C) and (39), (40),

$$
\left\|\phi^{1 / 2} \xi^{n+1}\right\|^{2}-\left\|\phi^{1 / 2} \xi^{n}\right\|^{2}+\left\|\nabla_{h} \xi^{n+1}\right\|^{2} \Delta t \leq M\left\{\left\|\xi^{n+1}\right\|^{2}+\left\|\xi^{n}\right\|^{2}+h^{4}+(\Delta t)^{2}\right\} \Delta t .
$$

Summing (41) on $t$ for $0 \leq n \leq L$, and noting that $\xi^{0}=0$,

$$
\left\|\phi^{1 / 2} \xi^{L+1}\right\|^{2}+\sum_{n=0}^{L}\left\|\nabla_{h} \xi^{n+1}\right\|^{2} \Delta t \leq M \sum_{n=0}^{L}\left\{\left\|\xi^{n+1}\right\|^{2} \Delta t+h^{4}+(\Delta t)^{2}\right\} \Delta t .
$$

Applying Gronwall lemma,

$$
\left\|\xi^{L+1}\right\|^{2}+\sum_{n=0}^{L}\left\|\xi^{n+1}\right\|_{1}^{2} \Delta t \leq M\left\{h^{4}+(\Delta t)^{2}\right\},
$$

where $\left\|\xi^{n+1}\right\|_{1}^{2}=\left\|\nabla_{h} \xi^{n+1}\right\|^{2}+\left\|\xi^{n+1}\right\|^{2}$.

It remains to verify the induction hypothesis (35). It is right as $n=0$ because of $\xi^{0}=0$. Assume the induction hypothesis holds for any positive integer $n$ between 1 and a given positive integer $l$. By (43) and (31) we have $\left\|\pi^{l+1}\right\|_{0}+\left\|\xi^{l+1}\right\|_{0} \leq M\left\{h^{2}+\Delta t\right\}$. The inequality $\left\|\pi^{l+1}\right\|_{0, \infty}+\left\|\xi^{l+1}\right\|_{0, \infty} \leq M h^{1 / 2}$ follows from (13) and the maximum norm estimates, and (35) holds for $n=l+1$. Therefore, we finish the proof of error estimate (28a).

It continues to discuss error estimates of the components concentration. An equivalent expression is given after cancelling $S_{\alpha}^{n+1 / 3}, S_{\alpha}^{n+2 / 3}$ from (23), (24) and (25) and introducing a new notation $\hat{\phi}^{n+1,-1}=\left(\hat{\phi}^{n+1}\right)^{-1}$,

$$
\begin{aligned}
& \hat{\phi}_{i j k}^{n+1} \frac{S_{\alpha, i j k}^{n+1}-\hat{S}_{\alpha, i j k}^{n}}{\Delta t}-\sum_{\beta=1}^{3} \delta_{\bar{x}_{\beta}}\left(\hat{D}_{\alpha}^{n+1} \delta_{x_{\beta}} S_{\alpha}^{n+1}\right)_{i j k} \\
& =Q_{\alpha}\left(S_{\alpha, i j k}^{n}\right)-S_{\alpha, i j k}^{n}\left(q\left(C_{i j k}^{n+1}\right)-\phi_{i j k} \frac{C_{i j k}^{n+1}-C_{i j k}^{n}}{\Delta t}\right) \\
& -\Delta t\left\{\delta_{\bar{x}_{1}}\left(\hat{D}_{\alpha}^{n+1} \delta_{x_{1}}\left(\hat{\phi}^{n+1,-1} \delta_{\bar{x}_{2}}\left(\hat{D}_{\alpha}^{n+1} \delta_{x_{2}} S_{\alpha}^{n+1}\right)\right)\right)_{i j k}+\cdots+\delta_{\bar{x}_{2}}\left(\hat{D}_{\alpha}^{n+1} \delta_{x_{2}}\left(\hat{\phi}^{n+1,-1} \delta_{\bar{x}_{3}}\left(\hat{D}_{\alpha}^{n+1} \delta_{x_{3}} S_{\alpha}^{n+1}\right)\right)\right)_{i j k}\right\} \\
& \left.+(\Delta t)^{2} \delta_{\bar{x}_{1}}\left(\hat{D}_{\alpha}^{n+1} \delta_{x_{1}} \delta_{\bar{x}_{2}}\left(\hat{D}_{\alpha}^{n+1} \delta_{x_{2}}\left(\hat{\phi}^{n+1,-1} \cdot \delta_{\bar{x}_{3}}\left(\hat{D}_{\alpha}^{n+1} \delta_{x_{3}} S_{\alpha}^{n+1}\right)\right)\right)\right)\right)_{i j k}, 1 \leq i, j, k \leq N, \alpha=1,2, \cdots, n_{c} .
\end{aligned}
$$

Collecting (10) $\left(t=t^{n+1}\right)$ and (44), and letting $\tilde{\phi}_{i j k}^{n+1}=\left(\phi c^{n+1}\right)_{i j k}, \tilde{D}_{\alpha, i j k}^{n+1}=\left(c^{n+1} \phi K_{\alpha}\right)_{i j k}$, we can derive the following 
error equation,

$$
\begin{aligned}
& \hat{\phi}_{i j k}^{n+1} \frac{\zeta_{\alpha, i j k}^{n+1}-\left(s_{\alpha}\left(\bar{X}_{i j k}^{n}\right)-\hat{S}_{\alpha, i j k}^{n}\right)}{\Delta t}-\sum_{\beta=1}^{3} \delta_{\bar{x}_{\beta}}\left(\tilde{D}_{\alpha}^{n+1} \delta_{x_{\beta}} \zeta_{\alpha}^{n+1}\right)_{i j k} \\
& =\left\{\left(\hat{\phi}^{n+1}-\tilde{\phi}^{n+1}\right) \frac{\partial s_{\alpha}^{n+1}}{\partial t}\right\}_{i j k}+Q_{\alpha}\left(C_{i j k}^{n+1}, S_{\alpha, i j k}^{n}\right)-Q_{\alpha}\left(c_{i j k}^{n+1}, s_{\alpha, i j k}^{n+1}\right) \\
& +\left\{\left(S_{\alpha}^{n} q\left(C^{n+1}\right)-s_{\alpha}^{n+1} q\left(c^{n+1}\right)\right)_{i j k}+\left(S_{\alpha}^{n} \phi \frac{C^{n+1}-C^{n}}{\Delta t}-s_{\alpha}^{n+1} \phi \frac{\partial c^{n+1}}{\partial t}\right)_{i j k}\right\} \\
& -\Delta t\left\{\delta_{\bar{x}_{1}}\left(\tilde{D}_{\alpha}^{n+1} \delta_{x_{1}}\left(\tilde{\phi}^{n+1,-1} \delta_{\bar{x}_{2}}\left(\tilde{D}_{\alpha}^{n+1} \delta_{x_{2}} s_{\alpha}^{n+1}\right)\right)\right)_{i j k}-\delta_{\bar{x}_{1}}\left(\hat{D}_{\alpha}^{n+1} \delta_{x_{1}}\left(\hat{\phi}^{n+1,-1} \delta_{\bar{x}_{2}}\left(\hat{D}_{\alpha}^{n+1} \delta_{x_{2}} S_{\alpha}^{n+1}\right)\right)\right)_{i j k}+\cdots\right\} \\
& +(\Delta t)^{2}\left\{\delta_{\bar{x}_{1}}\left(\tilde{D}_{\alpha}^{n+1} \delta_{x_{1}}\left(\tilde{\phi}^{n+1,-1} \delta_{\bar{x}_{2}}\left(\tilde{D}_{\alpha}^{n+1} \delta_{x_{2}}\left(\tilde{\phi}^{n+1,-1} \cdot \delta_{\bar{x}_{3}}\left(\tilde{D}_{\alpha}^{n+1} \delta_{x_{3}} s_{\alpha}^{n+1}\right)\right)\right)\right)\right)_{i j k}\right. \\
& \left.-\delta_{\bar{x}_{1}}\left(\hat{D}_{\alpha}^{n+1} \delta_{x_{1}}\left(\hat{\phi}^{n+1,-1} \cdot \delta_{\bar{x}_{2}}\left(\hat{D}_{\alpha}^{n+1} \delta_{x_{2}}\left(\hat{\phi}^{n+1,-1} \delta_{\bar{x}_{3}}\left(\hat{D}_{\alpha}^{n+1} \delta_{x_{3}} S_{\alpha}^{n+1}\right)\right)\right)\right)\right)_{i j k}\right\} \\
& +\varepsilon_{\alpha, i j k}, 1 \leq i, j, k \leq N-1, \alpha=1,2, \cdots, n_{c},
\end{aligned}
$$

where $\bar{X}_{i j k}^{n}=X_{i j k}-\tilde{\phi}_{i j k}^{n+1,-1} \mathbf{u}_{i j k}^{n+1} \Delta t,\left|\varepsilon_{\alpha, i j k}\right| \leq M\left\{h^{2}+\Delta t\right\}, \alpha=1,2, \cdots, n_{c}$.

In numerical analysis bound water almost exists everywhere in oil reservoir, that is to say there exists a positive number $c_{*}$ such that $c(X, t) \geq c_{*}>0$. Noticing the convergence result of $c(X, t)$, (43), we can obtain the inequality for $h$ and $\Delta t$ sufficiently small

$$
C(X, t) \geq \frac{c_{*}}{2} .
$$

Multiplying both sides of (45) by $\zeta_{\alpha, i j k}^{n+1} \Delta t$, making inner products, we can obtain

$$
\begin{aligned}
& \left\langle\hat{\phi}^{n+1} \frac{\zeta_{\alpha}^{n+1}-\zeta_{\alpha}^{n}}{\Delta t}, \zeta_{\alpha}^{n+1}\right\rangle \Delta t-\sum_{\beta=1}^{3}\left\langle\hat{D}_{\alpha}^{n+1} \delta_{x_{\beta}} \zeta_{\alpha}^{n+1}, \delta_{x_{\beta}} \zeta_{\alpha}^{n+1}\right\rangle \Delta t \\
& \leq\left\langle\hat{\phi}^{n+1} \frac{\hat{\zeta}_{\alpha}^{n}-\zeta_{\alpha}^{n}}{\Delta t}, \zeta_{\alpha}^{n+1}\right\rangle \Delta t+\left\langle\left(\hat{\phi}^{n+1}-\tilde{\phi}^{n+1}\right) \frac{\partial s_{\alpha}^{n+1}}{\partial t}, \zeta_{\alpha}^{n+1}\right\rangle \Delta t+\left\langle Q_{\alpha}\left(C^{n+1}, S_{\alpha}^{n}\right)-Q_{\alpha}\left(c^{n+1}, s_{\alpha}^{n+1}\right), \zeta_{\alpha}^{n+1}\right\rangle \Delta t \\
& +\left\langle S_{\alpha}^{n} q\left(C^{n+1}\right)-s_{\alpha}^{n+1} q\left(c^{n+1}\right), \zeta_{\alpha}^{n+1}\right\rangle \Delta t+\left\langle S_{\alpha}^{n} \phi \frac{C^{n+1}-C^{n}}{\Delta t}-s_{\alpha}^{n+1} \phi \frac{\partial c^{n+1}}{\partial t}, \zeta_{\alpha}^{n+1}\right\rangle \Delta t \\
& -\Delta t\left\{\left\langle\delta_{\bar{x}_{1}}\left(\tilde{D}_{\alpha}^{n+1} \delta_{x_{1}}\left(\tilde{\phi}^{n+1,-1} \delta_{\bar{x}_{2}}\left(\tilde{D}_{\alpha}^{n+1} \delta_{x_{2}} s_{\alpha}^{n+1}\right)\right)\right)-\delta_{\bar{x}_{1}}\left(\hat{D}_{\alpha}^{n+1} \delta_{x_{1}}\left(\hat{\phi}^{n+1,-1} \delta_{\bar{x}_{2}}\left(\hat{D}_{\alpha}^{n+1} \delta_{x_{2}} S_{\alpha}^{n+1}\right)\right)\right), \zeta_{\alpha}^{n+1}\right\rangle+\cdots\right\} \Delta t \\
& +(\Delta t)^{2}\left\langle\delta_{\bar{x}_{1}}\left(\tilde{D}_{\alpha}^{n+1} \delta_{x_{1}}\left(\tilde{\phi}^{n+1,-1} \delta_{\bar{x}_{2}}\left(\tilde{D}_{\alpha}^{n+1} \delta_{x_{2}}\left(\tilde{\phi}^{n+1,-1} \cdot \delta_{\bar{x}_{3}}\left(\tilde{D}_{\alpha}^{n+1} \delta_{x_{3}} s_{\alpha}^{n+1}\right)\right)\right)\right)\right)\right. \\
& \left.-\delta_{\bar{x}_{1}}\left(\hat{D}_{\alpha}^{n+1} \delta_{x_{1}}\left(\hat{\phi}^{n+1,-1} \cdot \delta_{\bar{x}_{2}}\left(\hat{D}_{\alpha}^{n+1} \delta_{x_{2}}\left(\hat{\phi}^{n+1,-1} \delta_{\bar{x}_{3}}\left(\hat{D}_{\alpha}^{n+1} \delta_{x_{3}} S_{\alpha}^{n+1}\right)\right)\right)\right)\right), \zeta_{\alpha}^{n+1}\right\rangle \Delta t+M\left\{h^{4}+(\Delta t)^{2}\right\} \Delta t .
\end{aligned}
$$

Using (28a), we give the estimates of the terms on the left-hand side of (47),

$$
\left\langle\hat{\phi}^{n+1} \frac{\zeta_{\alpha}^{n+1}-\zeta_{\alpha}^{n}}{\Delta t}, \zeta_{\alpha}^{n+1}\right\rangle \Delta t \geq \frac{1}{2}\left\{\left\langle\hat{\phi}^{n+1} \zeta_{\alpha}^{n+1}, \zeta_{\alpha}^{n+1}\right\rangle-\left\langle\hat{\phi}^{n} \zeta_{\alpha}^{n}, \zeta_{\alpha}^{n}\right\rangle\right\}-M\left\|\zeta_{\alpha}^{n}\right\|^{2} \Delta t
$$

Similarly, by using (28a), the terms on the right-hand side of (47) are estimated as follows:

$$
\begin{aligned}
& \left\langle\hat{\phi}^{n+1} \frac{\hat{\zeta}_{\alpha}^{n}-\zeta_{\alpha}^{n}}{\Delta t}, \zeta_{\alpha}^{n+1}\right\rangle \Delta t \leq \varepsilon\left\|\nabla_{h} \zeta_{\alpha}^{n}\right\|^{2} \Delta t+M\left\|\zeta_{\alpha}^{n+1}\right\|^{2} \Delta t, \\
& \left\langle\left(\hat{\phi}^{n+1}-\tilde{\phi}^{n+1}\right) \frac{\partial s_{\alpha}^{n+1}}{\partial t}, \zeta_{\alpha}^{n+1}\right\rangle \Delta t \leq M\left\{h^{4}+(\Delta t)^{2}+\left\|\zeta_{\alpha}^{n+1}\right\|^{2}\right\} \Delta t, \\
& \left\langle Q_{\alpha}\left(C^{n+1}, S_{\alpha}^{n}\right)-Q_{\alpha}\left(c^{n+1}, s_{\alpha}^{n+1}\right), \zeta_{\alpha}^{n+1}\right\rangle \Delta t+\left\langle S_{\alpha}^{n} q\left(C^{n+1}\right)-s_{\alpha}^{n+1} q\left(c^{n+1}\right), \zeta_{\alpha}^{n+1}\right\rangle \Delta t \\
& +\left\langle S_{\alpha}^{n} \phi \frac{C^{n+1}-C^{n}}{\Delta t}-s_{\alpha}^{n+1} \phi \frac{\partial c^{n+1}}{\partial t}, \zeta_{\alpha}^{n+1}\right\rangle \Delta t \leq M\left\{h^{4}+(\Delta t)^{2}+\left\|\zeta_{\alpha}^{n+1}\right\|^{2}+\left\|\zeta_{\alpha}^{n}\right\|^{2}\right\} \Delta t .
\end{aligned}
$$

In a similar analysis of (37) (40), the other terms on the right-hand side are estimated,

$$
\begin{aligned}
& -\Delta t\left\{\left\langle\delta_{\bar{x}_{1}}\left(\tilde{D}_{\alpha}^{n+1} \delta_{x_{1}}\left(\tilde{\phi}^{n+1,-1} \delta_{\bar{x}_{2}}\left(\tilde{D}_{\alpha}^{n+1} \delta_{x_{2}} s_{\alpha}^{n+1}\right)\right)\right)-\delta_{\bar{x}_{1}}\left(\hat{D}_{\alpha}^{n+1} \delta_{x_{1}}\left(\hat{\phi}^{n+1,-1} \delta_{\bar{x}_{2}}\left(\hat{D}_{\alpha}^{n+1} \delta_{x_{2}} S_{\alpha}^{n+1}\right)\right)\right), \zeta_{\alpha}^{n+1}\right\rangle+\cdots\right\} \Delta t \\
& \leq M\left\|\zeta_{\alpha}^{n+1}\right\|^{2} \Delta t,
\end{aligned}
$$




$$
\begin{aligned}
& (\Delta t)^{2}\left\langle\delta_{\bar{x}_{1}}\left(\tilde{D}_{\alpha}^{n+1} \delta_{x_{1}}\left(\tilde{\phi}^{n+1,-1} \delta_{\bar{x}_{2}}\left(\tilde{D}_{\alpha}^{n+1} \delta_{x_{2}}\left(\tilde{\phi}^{n+1,-1} \cdot \delta_{\bar{x}_{3}}\left(\tilde{D}_{\alpha}^{n+1} \delta_{x_{3}} s_{\alpha}^{n+1}\right)\right)\right)\right)\right)\right. \\
& \left.-\delta_{\bar{x}_{1}}\left(\hat{D}_{\alpha}^{n+1} \delta_{x_{1}}\left(\hat{\phi}^{n+1,-1} \cdot \delta_{\bar{x}_{2}}\left(\hat{D}_{\alpha}^{n+1} \delta_{x_{2}}\left(\hat{\phi}^{n+1,-1} \delta_{\bar{x}_{3}}\left(\hat{D}_{\alpha}^{n+1} \delta_{x_{3}} S_{\alpha}^{n+1}\right)\right)\right)\right)\right), \zeta_{\alpha}^{n+1}\right\rangle \Delta t \leq M\left\|\zeta_{\alpha}^{n+1}\right\|^{2} \Delta t,
\end{aligned}
$$

Substituting (48) and (49) respectively into the left-hand side and the right-hand side of (47), summing on $t$ from $n=0$ to $n=L$, and noting that $\zeta_{\alpha}^{0}=0$, we have

$$
\left\|\left(\hat{\phi}^{n+1}\right)^{1 / 2} \zeta_{\alpha}^{L+1}\right\|^{2}+\sum_{n=0}^{L} \sum_{\beta=1}^{3}\left\|\left(\hat{D}^{n+1}\right)^{1 / 2} \delta_{x_{\beta}} \zeta_{\alpha}^{n+1}\right\|^{2} \Delta t \leq M\left\{\sum_{n=0}^{L}\left\|\zeta_{\alpha}^{L+1}\right\|^{2} \Delta t+h^{4}+(\Delta t)^{2}\right\} .
$$

Applying Gronwall Lemma, we get

$$
\left\|\zeta_{\alpha}^{L+1}\right\|^{2}+\sum_{n=0}^{L}\left\|\nabla_{h} \zeta_{\alpha}^{n+1}\right\|^{2} \Delta t \leq M\left\{h^{4}+(\Delta t)^{2}\right\}, \alpha=1,2, \cdots, n_{c} .
$$

Then (28b) is proved.

\section{Actual Applications}

The implicit characteristic fractional step method has been applied successfully in software design of enhanced oil production of the polymer flooding and numerical simulation and analysis of actual oil production in Daqing Oilfield. The mathematical model is formulated as follows (Ewing, et al., 1988; Yuan, 2013; Yuan, et al., 1998) $1,2,3$ :

$$
\begin{aligned}
& \frac{\partial}{\partial x}\left[\lambda_{l}\left(\frac{\partial p_{l}}{\partial x}-\gamma_{l} \frac{\partial z}{\partial x}\right)\right]=\frac{\partial}{\partial t}\left(\phi \frac{s_{l}}{B_{l}}\right)-q_{l}, l=w, o, \\
& p_{c}=p_{o}-p_{w}, s_{w}+s_{o}=1 .
\end{aligned}
$$

Let " $w "$ and " $o "$ refer to the water and the oil, and let $\phi$ mean the porosity. The symbols of the $l$-th phase are defined as follows. $p_{l}$ denotes the pressure, $s_{l}$ means the saturation, $B_{l}$ means the volume factor, $\lambda_{l}$ denotes the fluidity, $\gamma_{l}$ is the proportion, $q_{l}$ is the source sink term, and $p_{c}$ is the pressure of capillary.

The mathematical model of the motion of the polymer, kation and anion components is described by a system of convection-diffusion equations,

$$
\frac{\partial}{\partial t}\left(\phi s_{w} c_{\alpha}\right)+\nabla\left(c_{\alpha} \mathbf{u}-\phi k_{\alpha} \nabla c_{\alpha}\right)=Q_{\alpha}, \alpha=1,2, \cdots, n_{c} .
$$

where $c_{\alpha}=c_{\alpha}(x, t)\left(\alpha=1,2, \cdots, n_{c}\right)$ denotes the concentration of $\alpha$-component, and $n_{c}$ is the number of components. The simplified model of (52) and (53) is turned into the system of (8) (13)(Ewing, et al., 1989; Yuan, 2013; Shen, et al., 2002).
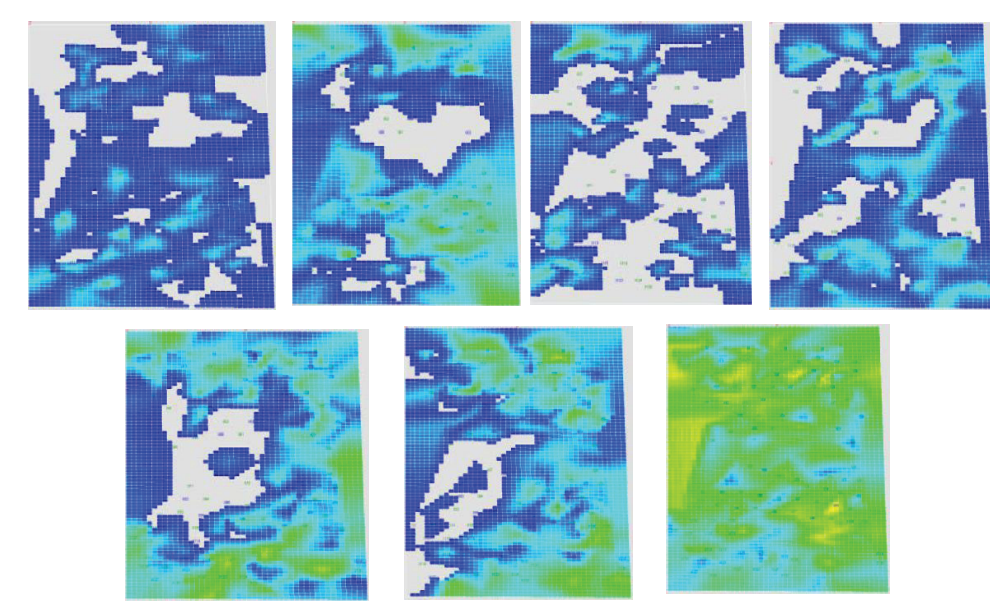

Figure 1. Effective thicknesses distribution of Xing Fourth Zone 


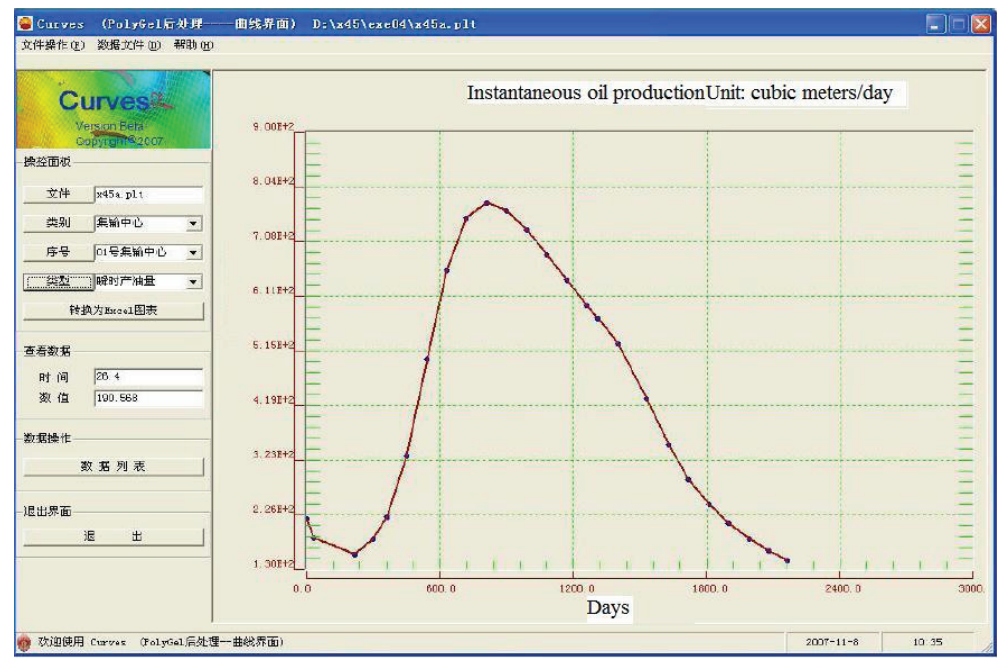

Figure 2. Sketch of instantaneous oil production curve of the polymer in Xing Fourth Zone

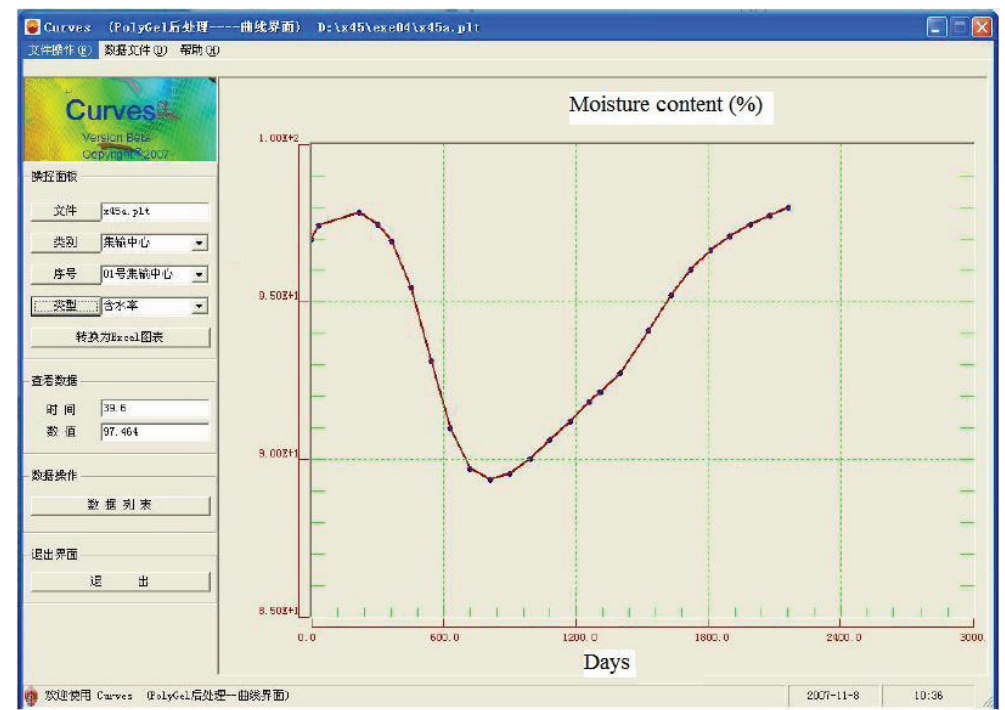

Figure 3. Sketch of water moisture curve of the polymer in Xing Fourth Zone

Table 1. Analysis of numerical data of matter balance

\begin{tabular}{|c|c|c|c||c|c|}
\hline \multirow{2}{*}{ Types of matter } & \multicolumn{5}{|c|}{ Components } \\
\cline { 2 - 6 } & Water & Oil & Polymer & Negion & Kation \\
\cline { 2 - 6 } & $\mathrm{SCM}$ & $\mathrm{SCM}$ & $\mathrm{Kg}$ & meq & meq \\
\hline Original content & $1.496 \mathrm{E}+7$ & $1.100 \mathrm{E}+7$ & $0.000 \mathrm{E}+0$ & $1.643 \mathrm{E}+11$ & $1.451 \mathrm{E}+11$ \\
\hline Injected quantity & $1.578 \mathrm{E}+7$ & $0.000 \mathrm{E}+0$ & $9.336 \mathrm{E}+6$ & $3.312 \mathrm{E}+11$ & $6.266 \mathrm{E}+10$ \\
\hline Produced quantity & $1.486 \mathrm{E}+7$ & $9.240 \mathrm{E}+5$ & $4.201 \mathrm{E}+6$ & $2.251 \mathrm{E}+11$ & $1.087 \mathrm{E}+11$ \\
\hline Current content & $1.588 \mathrm{E}+7$ & $1.008 \mathrm{E}+7$ & $5.135 \mathrm{E}+6$ & $2.703 \mathrm{E}+11$ & $9.913 \mathrm{E}+10$ \\
\hline Relative error & $3.602 \mathrm{E}-11$ & $1.219 \mathrm{E}-10$ & $2.853 \mathrm{E}-14$ & $6.905 \mathrm{E}-14$ & $3.510 \mathrm{E}-14$ \\
\hline
\end{tabular}

Experimental tests for Daqing Oilfield (Xing Fourth Zone of the polymer development area) are discussed by using 
the polymer flooding software. The three-dimensional region of geological model is decomposed into $46 \times 83 \times 7$ subdomains, and the effective thicknesses are distributed in Fig. 1. The sketch of instantaneous oil production and the curve of water moisture are shown in Fig. 2 and Fig. 3. Numerical data of matter balance are analyzed in Table 1. From this it is easy to see that numerical method of this type and its applicable software can keep high order of accuracy and can reflect correctly the physical process and the principle of polymer flooding. Main physical quantities distribute reasonably, computational accuracy is high, and some results of the polymer such as accumulation, endless loop don't arise.

Acknowledgements The authors express their deep appreciation to Prof. J. Douglas Jr, Prof. R. E. Ewing and Prof. L. S. Jiang for their many helpful suggestions in the serial of research on numerical simulation of enhanced (chemical) oil production.

\section{References}

Axelsson, O., \& Gustafasson, I. (1979). A modified upwind scheme for convective transport equations and the use of a conjugate gradient method for the solution of non-symmetric systems of equations. J. Inst. Maths. Applics., 23, 321-337. http://dx.doi.org/10.1093/imamat/23.3.321

Douglas, Jr. J., \& Gunn, J. E. (1963). Two order correct difference analogues for the equation of multidimensional heat flow. Math. Comp., 17(81), 71-80. http://dx.doi.org/10.1090/S0025-5718-1963-0149676-2

Douglas, Jr. J., \& Gunn, J. E. (1964). A general formulation of alternating direction methods, Part I. Parabolic and hyperbolic problems. Numer. Math., 6(5), 428-453. http://dx.doi.org/10.1007/BF01386093

Douglas, Jr. J. (1981). Simulation of miscible displacement in porous media by a modified method of characteristic procedure, Lecture Notes in Mathematics 912, Numerical Analysis, Proceedings, Dundee.

Douglas, Jr. J., \& Russell, T. F. (1982). Numerical method for convection-dominated diffusion problems based on combining the method of characteristics with finite element or finite difference procedures. SIAM J. Numer. Anal., 19(5), 871-885. http://dx.doi.org/10.1137/0719063

Douglas, Jr. J., \& Roberts, J. E. (1983). Numerical method for a model for compressible miscible displacement in porous media. Math. Comp., 4(164), 441-459. http://dx.doi.org/10.1090/S0025-5718-1983-0717695-3

Douglas, Jr. J. (1983). Finite difference methods for two-phase incompressible flow in porous media. SIAM. J. Numer. Anal., 20(4), 681-696. http://dx.doi.org/10.1137/0720046

Ewing, R. E. (1983). The Mathematics of Reservoir Simulation. SIAM, Philadelphia. http://dx.doi.org/10.1137/1.9781611971071

Ewing, R. E., Russell, T. F., \& Wheeler, M. F. (1984). Convergence analysis of an approximation of miscible displacement in porous media by mixed finite elements and a modified method of characteristics. Comp. Meth. Appl. Mech. Eng., 47(1-2), 73-92. http://dx.doi.org/10.1016/0045-7825(84)90048-3

Ewing, R. E., Yuan, Y. R., \& Li, G. (1989). Finite element for chemical-flooding simulation. Proceeding of the 7th International conference finite element method in flow problems, 1264-1271. The University of Alabama in Huntsville, Huntsville, Alabama: UAHDRESS.

Ewing, R. E., Lazarov, R. D., \& Vassilevski, A. T. (1994). Finite difference shceme for parabolic problems on composite grids with refinement in time and space. SIAM J.Numer. Anal., 31(6), 1605-1622. http://dx.doi.org/10.1137/0731083

Lazarov, R. D., Mishev, I. D., \& Vassilevski, P. S. (1996). Finite volume method for convection-diffusion problems. SIAM J. Numer. Anal., 33(1), 31-55. http://dx.doi.org/10.1137/0733003

Marchuk, G. I. (1990). Splitting and alternating direction method, In: Ciarlet, P.G., Lions, J. L., eds. Handbook of Numerical Analysis. Paris: Elsevior Science Publishers BV, 197-460. http://dx.doi.org/10.1016/S15708659(05)80035-3

Peaceman, D. W. (1980). Fundamental of Numerical Reservoir Simulation, Amsterdam: Elsevier.

Shen, P. P., Liu, M. X., \& Tang, L. (2002). Mathematical problems of petroleum exploration and development: Mathematical problems of oil-gas fields development, Part III. 197-264, Beijing: Science Press.

Yuan, Y. R. (1992). Time stepping along characteristics for the finite element approximation of compressible miscible displacement in porous media. Math. Numer. Sinica, 14(4), 386-406. 
Yuan, Y. R. (1993). Finite difference methods for a compressible miscible displacement problem in porous media. Math. Numer. Sinica, 15(1), 16-28.

Yuan, Y. R. (1994). Characteristic finite difference methods for moving boundary value problem of numerical simulation of oil deposit. Science in China, 37(A)12, 1442-1453.

Yuan, Y. R. (1996). The characteristic mixed-finite element method and analysis for three-dimensional moving boundary value problem. Science in China, 39(A)(3), $276 ł 288$.

Yuan, Y. R. (1996). Finite difference method and analysis for three-dimensional semiconductor device of heat conduction. Science in China, 39(A)11, 1140-1151.

Yuan, Y. R., Yang, D. P., Qi, L. Q., et al. (1998). Research on algorithms of applied software of the polymer. Qinlin Gang (editor in chief), Proceedings on chemical flooding, 246-253, Beijing: Petroleum Industry Press.

Yuan, Y. R. (2010). Theory and application of upwind finite difference method for moving boundary value problem of three-dimensional percolation coupled system. Science in China, 40(A) 2, 103-126.

Yuan, Y. R. (2012). The second-order upwind finite difference fractional step method for moving boundary value problem of nonlinear percolation coupled system. Science in China, 42(A) 8, 84-864.

Yuan, Y. R. (2013). Theory and application of numerical simulation of energy sources, basis of numerical simulation of chemical production (tertiary oil recovery). 257-304. Beijing: Science Press.

\section{Copyrights}

Copyright for this article is retained by the author(s), with first publication rights granted to the journal.

This is an open-access article distributed under the terms and conditions of the Creative Commons Attribution license (http://creativecommons.org/licenses/by/3.0/). 